far progressed as to permit passive motion. By the 17th of August the wound had entirely cicatrised, and the boy had complete use of his arm and joint.

\section{GLASGOW ROYAL INFIRMARY.}

\section{CASE OF ANEURISM OF THE FEMORAL AND POPLITEAL ARTERIES TREATED BY LIGATURE OF THE EXTERNAL ILIAC; RECOVERY.}

(Under the care of Dx. MORTON.)

THE following case is reported by Mr. S. Wellesley Coombs, surgeon's assistant.

Thomas $\mathrm{T}-$ - aged twenty-eight, a boiler-maker, was admitted with the following history. Four or five years ago a small swelling was observed just below Poupart's ligament on the right side. Three months before he hurt his left foot, and consequently had to use the right leg most. He then noticed that the lump began to get large, and continued to do so. Five weeks before admission the whole leg uniformly swelled, and was 4 in. larger than the left; but by rest in bed this rapidly subsided. His occupation compelled him to do heavy work in constrained positions. He had never had syphilis, and his general health was very good.

A tumour was situated in the upper and inner part of the thigh, near the centre, and immediately below Poupart's ligament. It measured $3 \mathrm{in}$. in each direction, and had a very strong distensile pulsation. A short sharp bruit was heard propagated downwards to the middle of the thigh, and the other aneurismal characteristics were well marked. In the centre of the popliteal space of the same limb there was another swelling, half the size of the former, with the same signs, except that a bruit was not audible. The rest of the arterial system showed no signs of disease.

On August 1st Dr. Morton tied the external iliac artery on the right side. This was done by the usual operation. The only noticeable feature was the presence of a large vein which crossed the artery and had to be held aside. The vessel was reached about one inch above its termination, and was here quite healthy. An ordinary silk ligature was passed around it, and the ends cut short. Pulsation in the tiumour and in the artery below immediately stopped. The wound was brought together by silver sutures, and a pad of lint and a bandage were applied; limb wrapped in cottonwool.

The after-treatment of the case may be described in a few words. The wound healed completely by first intention, and at the end of a week was quite sound. No pulsation returned in the tumours, and they gradually grew less. No pus formed, and the slight serous discharge was odourless. The ligature was not seen, but a small superficial sinus remained for a time near the centre of the wound, but this closed up before the patient left the hospital, which he did four weeks after the operation, quite well.

It may be remarked that after the first ten hours the temperature of the diseased limb was always a little higher than that of the sound member, but less than the axilla.

It may interest many to notice that the ligature remains enclosed, and that no antiseptic of any kind was used except the blood.

\section{Ittedural Soctetics.}

\section{PATHOLOGICAL SOCIETY OF LONDON.}

Spontaneous Cure of Aortic Aneurism.-Organs in Rabies.Lymphatics in Leprosy. - Early Addison's Disease.Defect in Septum of Ventricles.

THE ordinary meeting of this Society was held on the 5th inst., Dr. Murchison, President, in the chair. A discussion upon the "spontaneous cure" of large aneurisms, and a communication by Dr. Hoggan upon the changes in the lymphatics in leprosy were the chief subjects of interest.

Dr. RALFE showed a specimen of Thoracic Aneurism, spontaneously cured, from the body of a man admitted into the Seamen's Hospital last August. He had been previously in hospital with cardiac disease, and when readmitted was suffering from bronchitis and dropsy. There was a systolic murmur audible over the precordia. He died shortly after admission, and, on examination, the heart was found to be dilated and hypertrophied, weighing $13 \frac{1}{4} \mathrm{oz}$. , and the mitral valve was incompetent. The ascending aorta was the seat of a pouch, the size of the fist, which was filled up and obliterated by laminated fibrine, the whole clot weighing $7 \frac{3}{4} \mathrm{oz}$, the remaining lumen of the vessel containing a small post-mortem clot. There was ulceration of the inferior surface of the epiglottis, but not of the vocal cords; an elongated ragged cavity, containing pus, at the apex of the right lung, and a few scattered cretaceous deposits in both lungs, but no tubercle. The liver was congested and indurated, its capsule extensively thickened, whilst beneath the capsule of the spleen, and also beneath the capsule of each kidney, were numerous purulent foci, but no abscesses in the substance of these organs. Dr. Ralfe inferred that the patient was syphilitic. He was unable to say how the obliteration of the aneurism was started ; most of the arteries were atheromatous and of small size. Possibly the mitral incompetency aided in the consolidation. The pulmonary Iesion might be the result of pressure of the aneurism on the root of the lung, as Dr. Irvine had lately shown.

Dr. Douglas Powell showed a specimen analogous to Dr. Ralfe's. In this case, also of Thoracic Aneurism, the spontaneous cure was no doubt aided by the patient being kept absolutely at rest for about eleven weeks. He was thirty-one years of age, a cornet-player by profession, and had been under observation for about five years. $\mathrm{He}$ was admitted into the Brompton Hospital in October, 1575 and kept in the recumbent position, the only medicinal treatment being the administration of morphia to relieve pain. The physical sigus consisted of a double shock-sound and a slight systolic bruit, with an extensive area of dulness over the sternum. He left the hospital much improved, and remained in fairly good health for about two years, when, after failing in strength for some weeks, he slowly sank The specimen showed an enormous thoracic aneurism in. volving the root of the innominate, the whole sac being filled with laminated fibrin, a small channel only being left the left carotid artery was also partially occluded by clot, but the right carotid was not involved. Mr. Heath had been consulted as to the advisability of operative interference, but it was decided that no operation would have done any good, The lung in this case had not undergone any destructive change.

The PResident observed that the subject of spontaneous cure of aneurism was both of clinical and pathological in. terest. He remembered, when a student, seeing at Edin. burgh a patient under the care of the late Professor Bennett suffering from a large abdominal aneurism. It was treated on Valsalva's method, and after death was found to be nearly entirely consolidated; but there was also found a large aneurism in the chest, which had probably formed during the cure of the abdominal aneurism. - Dr. GREEY. How referred to a case which he had brought before the Clinical Society (see Clin. Soc. Trans., vol. ix., p. 109), where a large aneurism of the arch of the aorta was com. pletely filled with laminated fibrin, the spontaneous cure in this case having possibly been aided by the obliteration of the left carotid artery by the aneurismal sac. The patient died from the effects of an empyema in the left side. Mr. HULKE recalled two cases of aneurism which were under the care of the late Dr. Todd. They were both thoracic, and of large size, and were treated by recumbency and limited diet, with the result of great improvement in symptoms, recession of the tumour, and a cure which was apparently permanent. - Mr. ADAMS said there was a specimen in the museum of St. Thomas's Hospital of an aneurism involving the arch of the aorta and the large vessels, which was filled with laminated fibrine. He referred to the differences between rapid and gradual coagulation as evidenced by the clot being non-laminated, but uniform or fissured in a radiating manner, instead of being deposited in layers; and he believed that sometimes this coagulation led to the death of the patient, from pressure on the trachea being produced, or the tumour becoming consolidated. $\mathrm{He}$ thought this had occurred in the case of a well-known member of the profession for whom tracheotomy was performed with the result of prolonging the patient's life. In this case, on post-mortem examination, the aneurism-one involring the arch of the aorta, innominate and carotid arteries-was 